\title{
A simplified age-stage model for copepod population dynamics
}

\author{
Qiao Hu*, Cabell S. Davis, Colleen M. Petrik \\ Biology Department, Woods Hole Oceanographic Institution, Woods Hole, Massachusetts 02543, USA
}

\begin{abstract}
Complex 3D biological-physical models are becoming widely used in marine and freshwater ecology. These models are highly valued synthesizing tools because they provide insights into complex dynamics that are difficult to understand using purely empirical methods or theoretical analytical models. Of particular interest has been the incorporation of concentration-based copepod population dynamics into 3D physical transport models. These physical models typically have large numbers of grid points and therefore require a simplified biological model. However, concentrationbased copepod models have used a fine resolution age-stage structure to prevent artificially short generation times, known as numerical 'diffusion.' This increased resolution has precluded use of agestage structured copepod models in 3D physical models due to computational constraints. In this paper, we describe a new method, which tracks the mean age of each life stage instead of using age classes within each stage. We then compare this model to previous age-stage structured models. A probability model is developed with the molting rate derived from the mean age of the population and the probability density function (PDF) of molting. The effects of temperature and mortality on copepod population dynamics are also discussed. The mean-age method effectively removes the numerical diffusion problem and reproduces observed median development times (MDTs) without the need for a high-resolution age-stage structure. Thus, it is well-suited for finding solutions of concentration-based zooplankton models in complex biological-physical models.
\end{abstract}

KEY WORDS: Plankton · Copepods · Modeling · Marine ecology · Oceanography · Limnology · Methodology $\cdot$ Mean age

Resale or republication not permitted without written consent of the publishe

\section{INTRODUCTION}

Copepods are the dominant mesozooplankton in the ocean, accounting on average for $75 \%$ of the numbers and biomass collected in a $0.333 \mathrm{~mm}$ mesh plankton net (Longhurst et al. 1966). Copepods are also often dominant in freshwater systems, competing with cladocerans for resources. These organisms are consumers of primary producers and of protozoa in the microbial food web and are the dominant prey of many important animals, including fish larvae.

To quantify the complex underlying biologicalphysical mechanisms controlling abundance and distribution of copepods in aquatic environments, numerical modeling has proven to be an excellent tool (Runge et al. 2004). Many models have been devel- oped for quantifying copepod population dynamics, including stage-structured models (e.g. Wroblewski 1980, Gaedke 1990, Plagányi et al. 1999). A problem with these simplified structured copepod population models is 'temporal numerical diffusion.' This problem is due to insufficient resolution of the population structure on time scales of the temporal forcing, leading to artificially short generation times (Davis 1984a). To overcome this numerical diffusion problem, copepod population models have been developed that contain numerous age classes within each life stage (Davis 1984a,b, Sciandra 1986, Carlotti \& Sciandra 1989, Miller \& Tande 1993, Souissi \& Nival 1997).

Although the age-within-stage models significantly reduce or eliminate the numerical diffusion problem, the computational cost is relatively high. The number 
of age-classes within each developmental stage needs to be matched to the laboratory experimental results. The model is not likely transportable from one species to another. The number of age classes is dependent on the time step used in the model; thus, it cannot be greatly reduced without losing the biological resolution of the model. More recently, Gurney et al. (2001) proposed an alternative approach. They divided the whole life cycle into normalized classes. All physiological stages are then made up of multiple classes ( 2 to 10). They showed that their model can effectively eliminate numerical diffusion with fewer classes ( 100). Their approach is actually a special case of the agewithin-stage model. The ability to reduce the number of classes for their approach comes from ignoring individual variability. If we disregard individual variability in the age-within-stage model, many of the age classes will never be used and thus can be discarded. When we stack the age class into one age vector, we end up with the same model as Gurney et al. (2001) described.

Speirs et al. $(2004,2005)$ successfully applied the model of Gurney et al. (2001) to Calanus finmarchicus. They showed that they could further reduce the number of classes in their model by integrating the physical model. However, decreasing the number of state variables to reduce computational time came from a reduction of the spatial and temporal resolution. Nevertheless, their modeling results indirectly supported the idea that spatial numerical diffusion due to mixing and advection is less important than the temporal numerical diffusion.

Temporal numerical diffusion is a serious problem in copepod models and cannot be ignored (Souissi \& Ban 2001, Runge et al. 2004). Although age-within-stage models and their alternatives are able to reduce or eliminate the numerical diffusion problem, the use of such detailed age-stage structure is problematic when coupling it to 3D biological-physical models, which typically have very large numbers of 3D grid points (Runge et al. 2004). For example, combining a structured copepod model containing 200 age-stage classes (e.g. Davis 1984a) with a physical model containing $10^{6}$ grid points and a time step of a few minutes (e.g. FVCOM, Chen et al. 2006) leads to serious computational limitations on present day computers. We currently are modeling the 3D spatial patterns of dominant copepod species in the Gulf of Maine-Georges Bank region as part of the synthesis phase of the US GLOBEC program (GLOBEC 1992).

In testing various model formulations, we have created a new method for preventing artificial numerical diffusion in copepod models without the need to include age classes within each stage. This method simply uses the mean age of each life stage and can be applied to 13-stage models as well as aggregated mod- els (e.g. eggs-nauplii-copepodites-adults, ENCA). The following sections describe the problem of temporal numerical diffusion, the use of age classes within stages to prevent it, the formulation of the mean-agestage model, and a comparison of the new model to previous age-within-stage models. We also test the mean-age model with temperature-dependent molting and stage-dependent mortality.

\section{MODELS}

In this section, we define 3 kinds of population models that will be compared. First, the full age-withinstage model has a very detailed representation of the biological structure, resolving both the stage structure and the age distribution within each stage. Second, simplified life-stage models are developed using a traditional approach to group the copepod life cycle into major stages (ENCA and 13-stage model). Third, a simplified mean-age model only utilizing the mean age within each stage is developed.

Full age-within-stage model. We first describe the full age-within-stage model developed for the copepod Pseudocalanus sp. by Davis $(1984 \mathrm{a}, \mathrm{b})$. The state variable is $n_{i, k}$, i.e. the number of individuals that have been in stage $i$ for $k$ days. It evolves according to the following governing equations: molting,

$$
n_{i+1,0}(t+\mathrm{d} t)=\sum_{k=0}^{K_{i}} n_{i, \mathrm{k}}(t) S_{i} M_{i, \mathrm{k}}
$$

not molting,

$$
n_{i, k+1}(t+\mathrm{d} t)=n_{i, k}(t) S_{i}\left(1-M_{i, k}\right)
$$

new eggs produced,

$$
n_{1,0}(t+\mathrm{d} t)=\sum_{k=0}^{K_{13}} N_{13, k}(t) S_{13} F_{k}
$$

where $t$ is time (model time step $\mathrm{d} t$ is $1 \mathrm{~d}$ ), $M_{i, k}$ is the probability of molting from stage $i$ age $k$ to stage $i+1$ age 0 , determined from a cumulative normal distribution function with mean equal to the mean stage duration and standard deviation equal to $10 \%$ of the mean. $K_{i}$ is the number of age classes in stage $i$ and is 10 for each stage from 1 to 12 and 80 for adults, giving a total of 200 age-stage classes. The survival rate $S_{i}$ is assumed to be stage-dependent, while the egg production rate $F_{k}$ declines as the adults age. For demonstration purposes, the exact form of $F$ or $S$ is not critical, since we are focusing on the differences in the treatment of age-dependent molting rate between models. In principle, all of these rates may vary with time, but again, for demonstration purposes, we shall keep them fixed. The number molting to adult (i.e. the right hand 
side of Eq. 1 for $i=13$ ) was reduced by a factor of $1 / 2$ to represent a rapid die-off of males in order to approximate the observed adult female to male ratio in Pseudocalanus sp. of 10:1.

The full model given above can be rewritten into a population matrix form for any given age/stage:

$$
\mathbf{n}_{t+\mathrm{d} t}=\mathbf{L}_{t} \mathbf{n}_{t}
$$

where the population structure $\mathbf{n}$ is a vector that concatenates the various ages and stages into 1 column, and $\mathbf{L}_{\boldsymbol{t}}$ is a population transition matrix, which includes all the molting, mortality, and fertility coefficients.

Life-stage models: ENCA and 13-stage model. Solutions to the full model were compared to 2 simplified models to show the problems of numerical diffusion. In the simplest model (ENCA), individuals are grouped into 4 stages: eggs, nauplii, copepodites, and adults. An intermediate model containing all 13 life stages but no ages within stages also was examined. These models have the basic population matrix form:

$\left[\begin{array}{c}n_{1} \\ n_{2} \\ n_{3} \\ \vdots \\ n_{s}\end{array}\right]_{t+\mathrm{d} t}=\left[\begin{array}{cccc}\left(1-M_{1}\right) S_{1} & 0 & 0 & \cdots \\ M_{1} S_{1} & \left(1-M_{2}\right) S_{2} & 0 & \cdots \\ 0 & M_{2} S_{2} & \left(1-M_{3}\right) S_{3} & \ldots \\ \vdots & \vdots & \vdots & \ddots \\ 0 & 0 & \ldots & \frac{1}{2} M_{s-1} S_{s-1}\end{array}\right.$

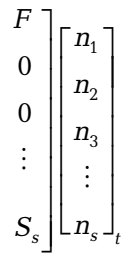

where $M_{i}$ and $S_{i}$ are probabilities of molting and survival, respectively, from stage $i$ to stage $i+1, s$ is the total number of life stages in the model, and $F$ is egg production rate per female (i.e. fertility).

Mean-age model. Simplification of the full model to the mean-age model involves 2 major steps. First, instead of tracking numerous age classes within each stage, only the mean age of the population within each stage is used. Then, probabilistic density functions are developed to model the molting process, and the mean age of each stage is used to index these probabilistic molting functions. The mean age of the population in each stage, $i$, is updated as follows.

If $i=1$,

$$
k_{1, t+\mathrm{d} t}=\frac{n_{1, t}\left[1-M_{1}\left(k_{1, t}\right)\right] S_{1}\left(k_{1, t}+1\right)}{e_{t+\mathrm{d} t}+n_{1, t} S_{1}\left[1-M_{1}\left(k_{1, t}\right)\right]}
$$

otherwise,

$$
k_{i, t+\mathrm{d} t}=\frac{n_{i, t}\left[1-M_{i}\left(k_{i, t}\right)\right] S_{i}\left(k_{i, t}+1\right)}{n_{i-1, t} M_{i-1}\left(k_{i-1, t}\right)+n_{i, t}\left[1-M_{i}\left(k_{i, t}\right)\right] S_{i}}
$$

Here $k_{i, t}$ is the mean age of the population in the $i$ th stage at time $t, e_{t+\mathrm{d} t}$ is the number of new eggs produced at time $t+\mathrm{d} t, n_{i, t}$ is the number of individuals in the $i$ th stage at time $t$, and $M_{i}\left(k_{i, t}\right)$ is the molting rate of the individuals from stage $i$, mean age $k_{i, t}$ to stage $i+1$, mean age 0 . The molting rate $M_{i}\left(k_{i, t}\right)$ is obtained from a simplified probability model. Assuming the probability density function (PDF) of molting at stage $i$ is $p_{i}(k)$ and its cumulative function is $P_{i}(k)$, the corrected molting rate $M_{i}(k)$ can be calculated as (Hu et al. 2007)

$$
M_{i}(k)=p_{i}(k) \mathrm{d} t /\left[1-P_{i}(k)\right]
$$

This corrected molting rate $M_{i}(k)$ is a function of age $k$, and is monotonically increasing. The mean age $k_{i, t}$ is used to index the molting rate $M_{i}(k)$ to obtain the molting rate of individuals in the ith stage of mean age $k$ at time $t$. We approximated the PDFs, $p_{i}(k)$, with piecewise linear functions, which allowed us to easily and quickly calculate the corrected molting rate function $M_{i}(k)$. The only parameters used to model the PDFs were the mean stage durations and their standard deviations.

\section{SIMULATIONS}

\section{The numerical diffusion problem: an example}

The effect of numerical diffusion in copepod models is easily seen by comparing numerical solutions of a 4-stage model (ENCA), a 13-stage model, and a 200-age-stage model, each having the same mean stage durations (Fig. 1). All models were started with 100 eggs at $t=1$ and evolved according to the molting rates calculated from the mean stage durations. The molting rates of both the ENCA and the 13-stage models were fixed for each stage, and they were inversely proportional to the mean stage duration. The molting rate formulation in the 200 age-within-stage model is given in Eq. $(1 \mathrm{a}, \mathrm{b})$.

This type of numerical diffusion results because the biological model has no metric to keep track of the age of the cohort. The molting rate is set constant and is usually the reciprocal of the median stage duration time. As a result, a small portion of animals will molt much earlier than biologically possible. On the other hand, a significant portion will remain in one stage much longer than the median stage duration time. The portion of animals with artificially shortened durations has received more attention because it will greatly shorten the turnover time in multiple generations. The effect of animals with lengthened durations has not been given much attention because most of these animals die before reaching the adult stage.

Both the ENCA and the 13-stage models had substantial early molting, which is seen in the leading edge of the curve for each life stage compared to the corresponding curves for the 200 age-within-stage model (Fig. 1). There is also significant delayed molting in the simpler models as evidenced by the trailing 

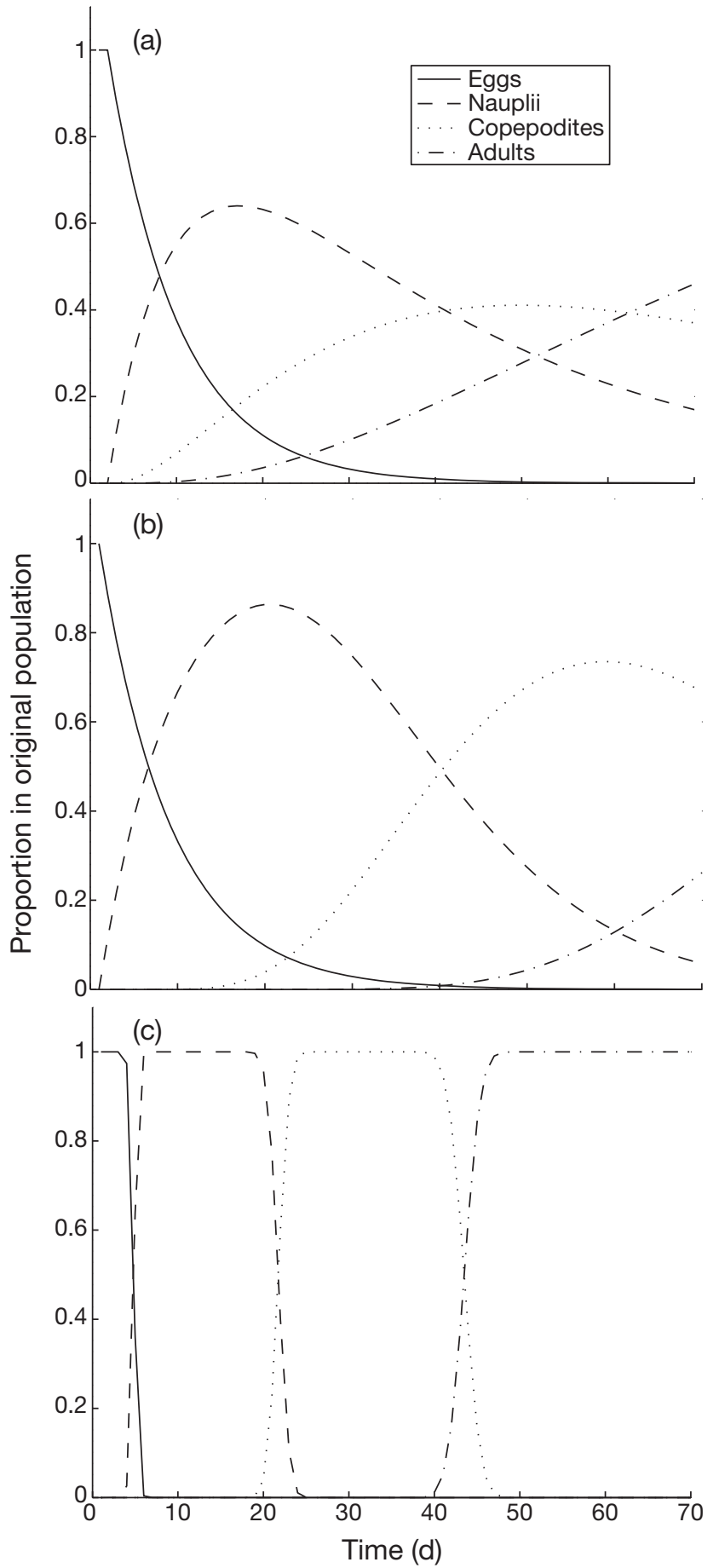

Fig. 1. Simulated populations using (a) an ENCA (eggs-nauplii-copepodites-adults) model, (b) a 13-stage model, and (c) a full age-within-stage model that were grouped into eggs $(-)$, nauplii $(---)$, copepodites I-V $(\cdots \cdots \cdots)$, and adults $(\cdot-\cdot)$ stages for plotting

edge of the curves for each life stage (Fig. 1). These effects are the result of the numerical diffusion caused by insufficient resolution in the age-stage structure.

\section{Grouping the life stages as a process of convolution}

Souissi \& Ban (2001) examined the effect of aggregating life stages in copepod population dynamics models. They suggested that the difference between the aggregated stage model and a finer resolution stage model was due to the difference in mortality among stages. However, we found that such a discrepancy can be explained by molting alone. Since the effect of grouping 2 life stages into 1 life stage in a model is equivalent to the convolution of the PDFs of these 2 stages, the resulting PDF can be used as the molting function for the combined stage (Bertsekas \& Tsitsiklis 2002).

We examined the PDFs from Souissi \& Ban (2001, their Table I, EXP1) and computed the convolutions. Ideally, the convolution of the 2 PDFs for N1 to N3 and N4 to N6 should be equal to the experimentally observed PDF for N1 to N6, because they describe the same random process. In practice, these curves can differ for 2 reasons. First, due to the small number of individuals monitored in the laboratory experiments, both sampling error and parameter fitting error could cause the difference between these 2 PDFs. Second, due to the nature of experiments in which copepods are individually reared, all PDFs of molting for different stages are estimated from the same individuals raised from eggs to adults. Thus, the PDFs of the different stages are not independent. However, in the population model, the PDFs are assumed to be independent.

In order to explain the disagreement in the 2 model results of Souissi \& Ban (2001, their Fig. 7), we compared the difference between 2 PDFs derived from their data (Fig. 2a,b). The solid and dot-dashed lines in Fig. 2a,b correspond to the solid and circled abundance curves, respectively, in Fig. 7B of Souissi \& Ban (2001). The leading edges of the PDFs for N1 to N6 and $\mathrm{C} 1$ to $\mathrm{C} 5$ rise earlier in the aggregated models than in the more detailed ones (Figs. 2a,b), explaining why the abundances of nauplii and copepodites in the more detailed model of Souissi \& Ban (2001, their Fig. 7B, circled curves $\mathrm{SG}_{1}, \mathrm{SG}_{2}$ ) decrease later than in their aggregated model (their solid curves). This causes the abundance of copepodites and adults in their more detailed model to increase later than in their aggregated model.

The difference in copepod molting PDFs between aggregated and detailed models can therefore explain the difference in the model results of Souissi \& Ban (2001), without the need for including a stage-specific mortality effect. Mortality can also contribute to disagreement between detailed and aggregate models, but we found this effect was small (see below). 


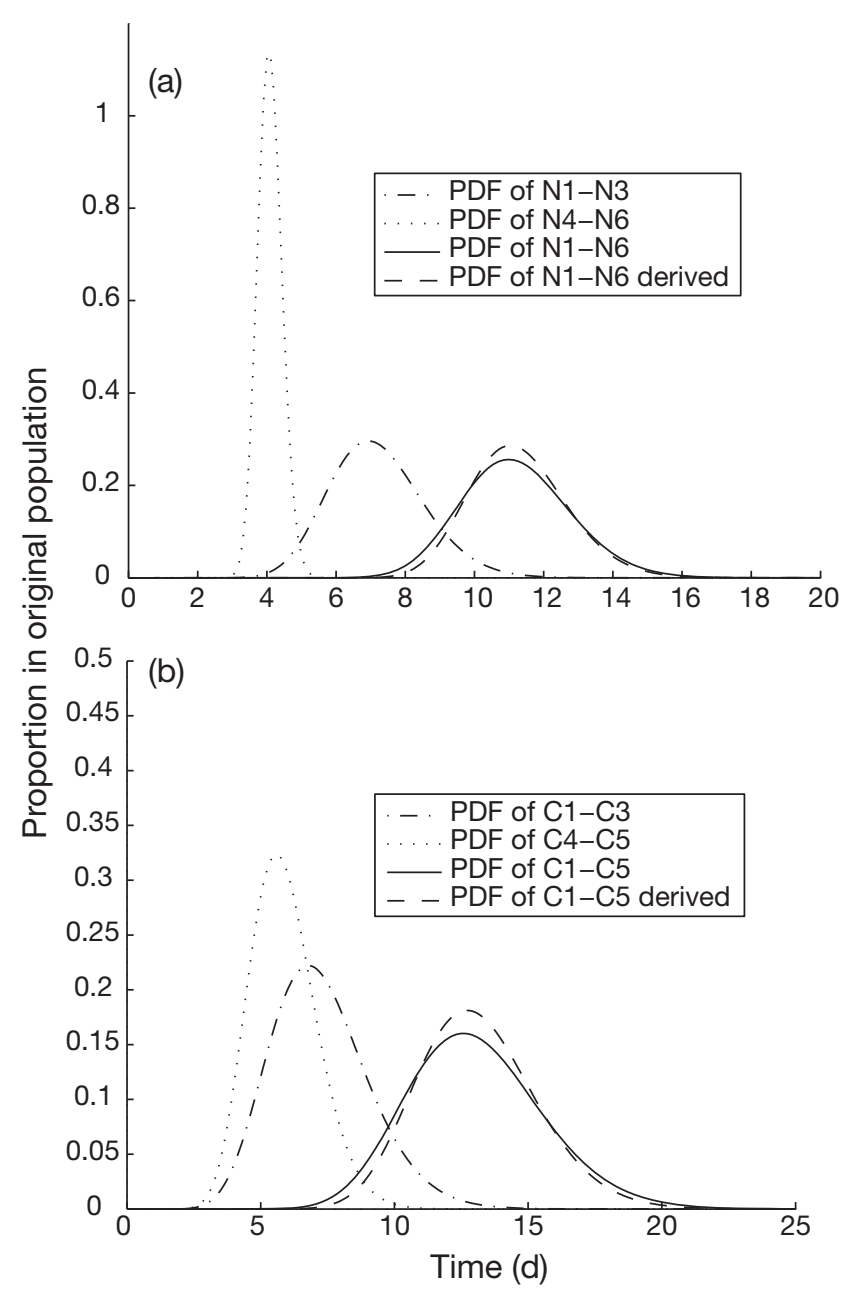

Fig. 2. Molting PDFs of (a) nauplii and (b) copepodites. With the exception of the derived PDF, the data were taken from Souissi \& Ban (2001). The dash-dotted and dotted lines are the molting PDFs of life stages N1-N3 / C1-C3 and N4-N6 / $\mathrm{C} 4-\mathrm{C} 5$, respectively, from the laboratory experiment. The convolution of these 2 PDFs is shown as the dashed line at right. The solid line is the PDF for copepod stage N1-N6 / C1-C5 from the laboratory experiment

\section{Molting PDFs in the population model}

Although there have been numerous laboratory experiments conducted on different copepod species at different temperatures and food conditions, the correct formulation of the molting rate for use in population models has only recently been found ( $\mathrm{Hu}$ et al. 2007). The main problem has been in applying the molting PDF estimated from laboratory experiments to population models. Models require probabilities of molting for the remaining population, while laboratory-derived molting PDFs are based on the initial population. Hu et al. (2007) proposed the following formula for the molting rate in the population model:

$$
f_{r}(t)=f_{o}(t) /\left(1-\int_{-\infty}^{t} f_{o}(x) \mathrm{d} x\right)
$$

where $f_{o}(t)$ is the PDF from the laboratory experiments, and $f_{r}(t)$ is the probability of molting for animals remaining in a given life stage at time $t$ (termed PMR by Hu et al. 2007)

\section{Mean-age model}

The main problem with stage-structured population models (e.g. ENCA or 13-stage) has been that agewithin-stage was not considered. Without age information, the PDF cannot be used to correctly model copepod molting rate. Instead, a fixed molting rate has been used, which causes numerical diffusion, resulting in artificially rapid development. The full age-within-stage model, on the other hand, requires so many state variables that it is not suitable for use in large 2D or 3D physical models. We solved this dilemma by developing a model that tracks the mean age of animals in each stage without the need for age classes within the stages. The mean age of each stage enabled us to use a simple PDF to compute the molting rate for each stage. We applied this approach to both ENCA and 13-stage models and present a comparison of the 4-stage (ENCA) mean-age model with the 200 age-within-stage model.

As discussed above, the molting PDF in the 200 agewithin-stage model had a normal distribution with a standard deviation of $10 \%$ of the mean. For simplicity, the molting PDF in the ENCA-mean-age model had a uniform distribution. Both models used the corrected molting function, PMR, in $\mathrm{Hu}$ et al. (2007). The agestructure in the full model and the mean age in the ENCA-mean-age model were used as indices in their corresponding PMRs to calculate the appropriate molting rate at each time step.

The life-stage curves for the ENCA-mean-age model were very similar to those produced by the 200 agewithin-stage model (Fig. 3). Comparing the solution of the mean-age-ENCA model to that of the ENCA model (Fig. 1a) reveals that the mean-age formulation removes the effects of numerical diffusion almost entirely. This result is remarkable considering that the ENCAmean-age model only has 8 state variables (number of individuals and mean age in each of the 4 stages) compared to the 200 state variables in the age-within-stage model. The mean-age model had a slightly shorter median development time (MDT) than the age-withinstage model. However, this difference is well within natural variation or laboratory experimental error. Table 1 summarizes the MDT of the model input (estimated from laboratory data) and the MDT of the model output. The difference in the MDT from egg to adult was less than $3.5 \mathrm{~d}$ among different models. Moreover, 


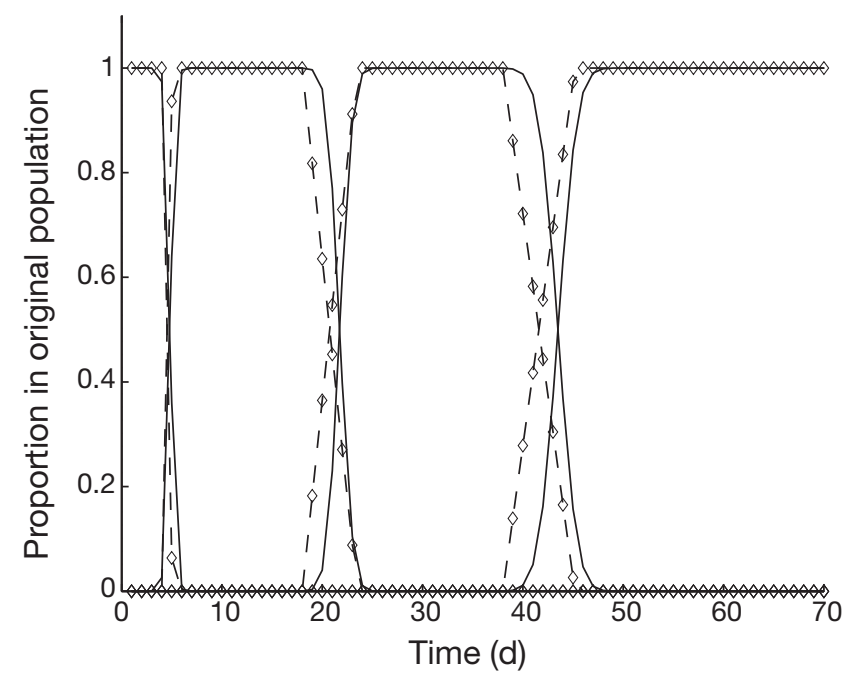

Fig. 3. Comparison results from the age-within-stage model $(-)$ and the ENCA-mean-age model (-- and $\diamond)$. The time step is $1 \mathrm{~d}$, temperature $\mathrm{T}=7.5^{\circ} \mathrm{C}$, and mortality $=0$. The curve for the age-within-stage model is identical to Fig. 1c, with the 4 peaks corresponding to eggs, nauplii, copepodites, and adults (i.e. 'ENCA')

this error in MDT estimation by the mean-age model came mainly from the fairly large time-step used (1 d). We show later in this section that with a smaller time step in the model, the MDT can be predicted even more precisely (Table 1).

\section{Mean-age model with mortality and varying temperature}

We tested the robustness of the ENCA-mean-age model to variations in internal parameters by allowing temperature to vary in time and mortality to vary with life stage (Fig. 4). Again, the numerical solutions of the ENCA-mean-age model were compared to the agewithin-stage model, using stage-dependent mortality and an annual sinusoidal variation in temperature:

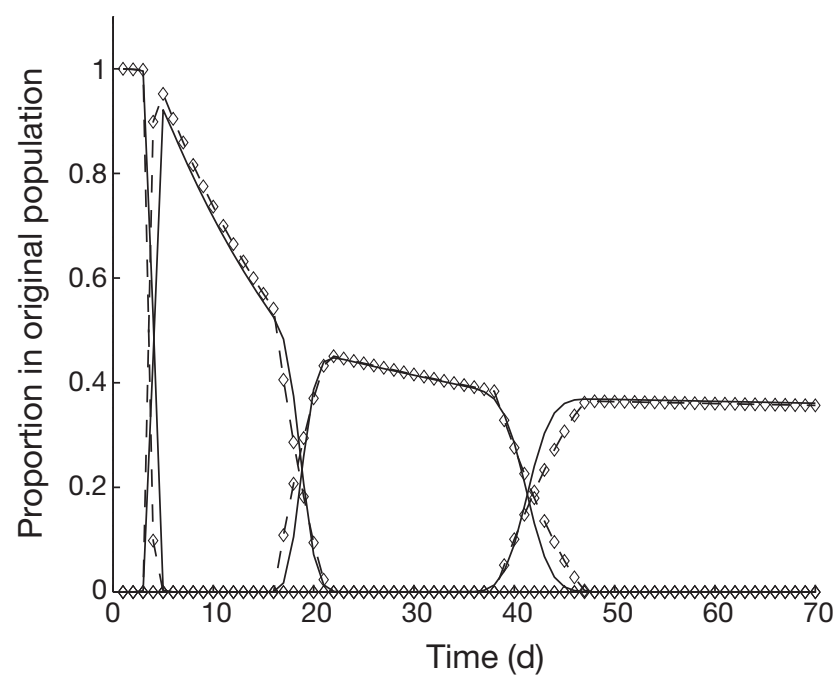

Fig. 4. Comparison of ENCA-mean-age $(---$ and $\diamond)$ and agewithin-stage (-) models, with varying temperature and stage specific mortality. The time step is $1 \mathrm{~d}$, temperature varies as sinusoidal function (Eq. 7). The mortalities are $0.001,0.05,0.01$, and 0.001 for eggs, nauplii, copepodites, and adults, respectively

$$
T(t)=5.7 \sin \left(\pi+\frac{2 \pi}{365} t\right)+10
$$

Mortality of eggs, nauplii, copepodites I-V, and adults were chosen as $0.1,5,1$, and $0.1 \%$ respectively (Davis 1984a,b). The ENCA-mean-age model was found to successfully approximate the 200 agewithin-stage model, even with addition of stage-specific mortality and time-varying temperature (Fig. 4). The maximum difference of MDT between these 2 models was less than $1 \mathrm{~d}$. At Day 50, the proportion of adults was $36.38 \%$ and $36.82 \%$ for ENCA-mean age and full age-stage models, respectively. The relative difference in population size over 1 generation between the 2 models was only $1.2 \%$.
Table 1. Median development times (MDT) of copepodite Pseudocalanus sp. The expected values were obtained from laboratory-based estimates reported by Davis $(1984 \mathrm{a}, \mathrm{b})$. TS: time step used in the model. Uniform probability density functions were used in all mean-age models. The MDTs were estimated as the time when $50 \%$ of the cumulative population had passed a given stage

\begin{tabular}{|lccccc|}
\hline $\begin{array}{l}\text { Life } \\
\text { stage }\end{array}$ & Expected & $\begin{array}{c}\text { Age- } \\
\text { within- } \\
\text { stage model } \\
(\mathrm{TS}=1 \mathrm{~d})\end{array}$ & $\begin{array}{c}\text { ENCA- } \\
\text { mean- } \\
\text { age model } \\
(\mathrm{TS}=1 \mathrm{~d})\end{array}$ & $\begin{array}{c}\text { 13-stage } \\
\text { mean- } \\
\text { age model } \\
(\mathrm{TS}=1 \mathrm{~d})\end{array}$ & $\begin{array}{c}\text { 13-stage } \\
\text { mean- } \\
\text { age model } \\
(\mathrm{TS}=0.1 \mathrm{~d})\end{array}$ \\
\hline Egg & 4.34 & 4.58 & 4.53 & 4.66 & 4.37 \\
N1-N6 & 20.99 & 21.48 & 20.71 & 19.74 & 21.85 \\
C1-C5 & 42.71 & 43.08 & 41.21 & 39.71 & 42.48 \\
\hline
\end{tabular}

\section{Effect of variable egg input}

We examined the response of the ENCA-mean-age model to a gradual input of the initial population of eggs (instead of starting with 100 eggs at $t=0$ ). The input of eggs was normally distributed according to the following functions:

$$
\begin{gathered}
n_{\text {egg }}=15 \mathrm{e}^{-(t-8)^{2} / 18} \\
n_{\text {egg }}=15 \mathrm{e}^{-(t-8)^{2} / 18}+15 \mathrm{e}^{-(t-38)^{2} / 18}
\end{gathered}
$$


where $t$ is time in days. The maximum rate of egg input was 15 eggs d $^{-1}$ at Day 8 in Eq. (8a). There were 2 peaks $30 \mathrm{~d}$ apart in Eq. (8b). Each peak had a maximum rate of egg input of 15 eggs d $^{-1}$ at Days 8 and 38. The responses of the ENCA-mean-age model and the agewithin-stage model were very close to each other (Fig. 5a,b). For the single-peak egg input (Fig. 5a), the maximal difference in MDT is less than $2.5 \mathrm{~d}$. Although the maximal difference in MDT is more than $10 \mathrm{~d}$ for the double-peak egg input case (Fig. 5b), the meanage model still captures the 2 cohorts very well. Because the abundance curve of the age-within-stage model around MDT is rather flat, we believe the MDT is not the best indicator to compare these 2 models. The ENCA-mean-age model had a much longer duration compared to single egg input (Fig. 4), which was caused by mixing new eggs into eggs with a much

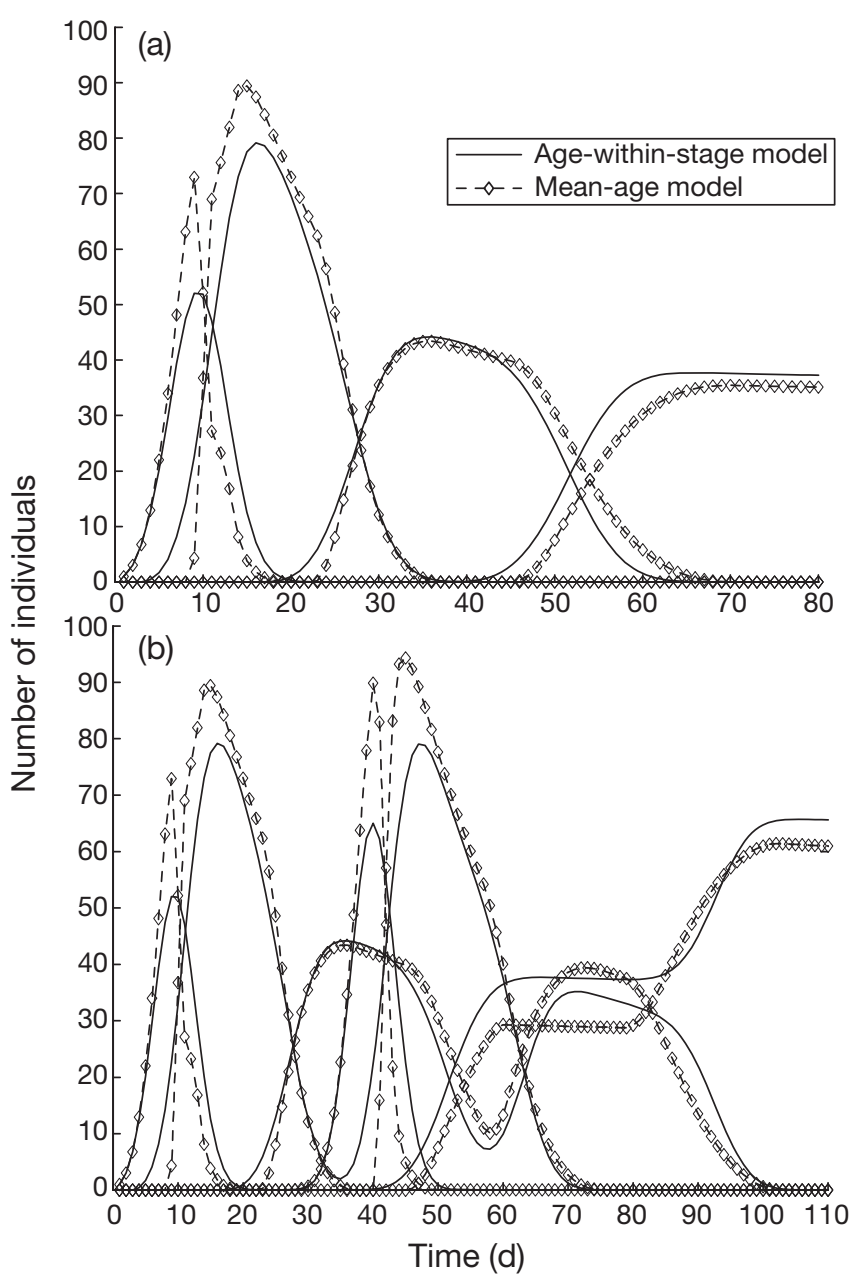

Fig. 5. Comparison of ENCA-mean-age (--- and $\diamond)$ and agewithin-stage (-) models, with varying temperature, stage specific mortality, and egg production turned on. The time step is $1 \mathrm{~d}$, temperature and mortalities used are the same as in Fig. 4. (a) Egg production varies as a Gaussian function (Eq. 8a), and (b) egg production varies as mixture of 2 Gaussian functions (Eq. 8b) older mean age, thus reducing the mean age of all eggs. It is more evident that the time of first molting was delayed substantially in the mean-age model, which could be seen from the much higher maximum egg abundance and the much later arrival time of the first nauplii (cf. Figs. 4 \& 5a,b). Nevertheless, this mixing effect on the overall performance of the mean-age model was not significant. For the single-peak egg input, at Day 72, the adults were 35.39 and 37.52 for ENCA-mean-age and full age-stage models, respectively. The relative difference in population size over 1 generation between the 2 models is less than $6 \%$ (Fig. 5a). If this holds for multiple generations, the relative difference over 5 generations is less than $27 \%$. Likewise, for the double-peak egg input, the relative difference in the population size at Day 110 between these 2 models is $7.4 \%$ (Fig. 5b). If this holds for multiple generations, the relative difference over 3 generations is $<22 \%$.

\section{More complicated PDFs}

We also investigated the difference between a simple PDF (uniform) and a complex PDF (normal) in the ENCA-mean-age model (Fig. 6). The time step used in these models was $1 \mathrm{~d}$. We can see clearly that the MDT predicted from the models was very close to estimates from laboratory experiments (cf. Table 1). There were only subtle differences between the normal distribution and the uniform distribution models at the leading and trailing edge of the moving cohort (Fig. 6). For the purpose of modeling population dynamics, we found a simple PDF to be sufficient and much faster to compute.

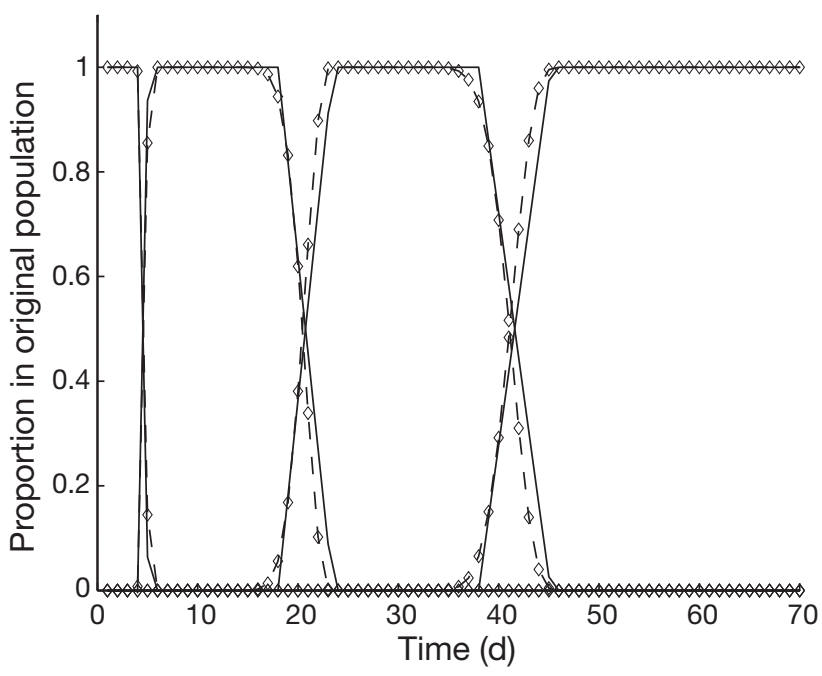

Fig. 6. Simulated populations using the ENCA-mean-age model with uniform (-) and normal (- - ) PDFs for the molting rates. The time step was $1 \mathrm{~d}$ 


\section{Time step of the mean-age model}

The time step in the mean-age model plays an important role in modeling development in the population dynamics model. We cannot use a fine time step in the difference equation for the full age-within-age model because it will introduce too many state variables. For example, halving the time step in the 200 age-within-stage model yields 400 age-stage classes. However, in the mean-age model, since we only tracked the mean age of each stage, using a fine time step did not introduce any extra state variables. The time step problem is more pronounced in a model with more life stages than it is in an aggregated model because the difference between the median stage duration (MSD) and time step becomes smaller. In an extreme case, when the MSD is less than or close to the time step, the error in MSD is unavoidable. Usually, the error in MSD is less than 1 time step for each developmental stage. We compared the 13-stage mean-age model with a time step of $1 \mathrm{~d}$ to the same model with a time step of $0.1 \mathrm{~d}$ (Fig. 7). We found the fine time step model had a more accurate MDT (cf. Table 1). Since the time step in coupled biological-physical models is usually $<<1 \mathrm{~d}$, the mean-age model provides an excellent approximation to the age-within-stage model without the associated memory and computational cost.

\section{DISCUSSION}

We developed a mean-age formulation that largely eliminates the effect of numerical diffusion in copepod population models, while increasing the number of

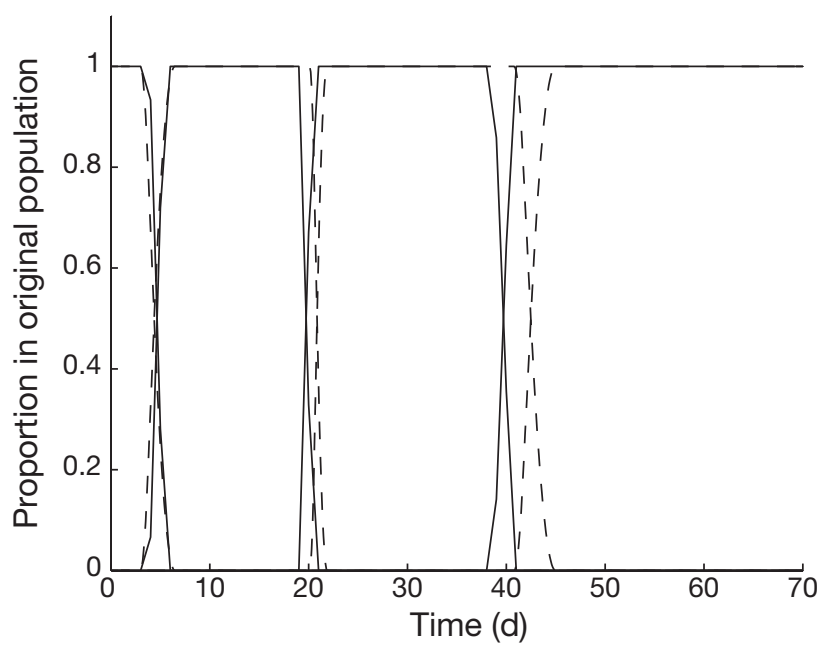

Fig. 7. Simulated populations using the 13-stage mean-age model with time steps of $0.1 \mathrm{~d}(-)$ and $1 \mathrm{~d}(---)$ state variables only by a factor of 2 over stage-only models. The mean-age formulation is significant, because it allows concentration-based copepod models to be used in 3D physical models without the large inaccuracies caused by numerical diffusion. Previously, age-within-stage models have been used to prevent numerical diffusion, but the number of state variables in these models is over an order of magnitude greater than in the corresponding stage-only models, precluding their use in 3D physical models for computational reasons.

In this paper, we assumed that the MSDs were known from the laboratory experiments and that they were accurate. Our model was able to predict these times using a very simple molting PDF. It was previously thought that a more complicated PDF was necessary to model the molting rate (e.g. gamma distribution; Soussi \& Ban 2001). A simple uniform PDF, in combination with the mean-age formulation, yields an accurate yet simplified concentration-based copepod population model.

We found that the grouping of intermediate stages was equivalent to the mathematical convolution of their molting PDFs. We demonstrated that such a model was not sensitive to important variations in the population dynamics, such as temperature-dependent development, stage-specific mortality, and temporally varying input to the population. Moreover, by tracking the mean-age of each stage, it is possible to incorporate age-dependent mortality into the model instead of using fixed stage-specific mortality as previous stageonly models have done.

The final result is an accurate copepod population model that is simple enough to use in spatially complex 3D biological-physical models. We are presently incorporating this model into a 3D biological-physical model of dominant copepod species in the Georges Bank-Gulf of Maine region as part of the US GLOBEC Northwest Atlantic program. More generally, the simple formulation presented here will allow broader use of concentration-based copepod models and their incorporation into physical models.

The main error source of the mean-age model compared to the age-within-stage model comes from mixing 2 different population structures. Due to the nature of the mean-age model, only the mean age of each developmental stage is recorded. When 2 populations with very different age structures get mixed, the mean-age model will make the younger group older than it is and the older group younger than it is. Fig. $5 \mathrm{a}$, b clearly illustrates such an effect. The performance of the mean-age model in these cases is not as good as in the cases of a single cohort. Fortunately, the mixing problem will not be so severe in the real ocean environment for the following 2 reasons: (1) the popu- 
lation structures being mixed in the neighboring water are very similar; or (2) the populations being mixed are totally unbalanced. The modeling work by Gurney et al. (2001) supports our argument because they showed that the physical model could be integrated into a very coarse grid but still yield a reasonable approximation. We are coupling this mean-age biological model with a 3D physical model. We did not find that the mixing and advection caused any problems in the coupled model. A detailed analysis of error due to mixing and advection in the coupled model is the subject of future research.

One reason individual based models (IBMs) of copepods have been so widely used is that they can be easily coupled to a 3D transport model with minimal computational cost, whereas the concentration-based models (CBMs) for copepods cannot. The simple mean-age copepod CBM developed here will allow wider use. CBMs of copepods can be naturally coupled with standard CBMs of food webs, such as the nutrient-phytoplankton-zooplankton-detritus (NPZD) class of models, since both CBMs exist at the same model grid points. In addition, CBMs of copepods allow for reproduction, whereas IBMs have to reproduce more particles, which can cause computational limitations. On the other hand, IBMs can include important details that cannot be easily be incorporated in CBMs (e.g. weight, lipid content, genetics). IBMs are necessarily sparse, with significant gaps developing during model runs, whereas the CBMs are continuous and can be better for computing budgets and determining, for example, whether a population is self-sustaining in a given region. The combination of these 2 kinds of modeling tools provides a complementary approach for studying the processes controlling copepod species in the ocean.

Acknowledgements. This work was supported by US GLOBEC NOAA grant NA17RJ1223.

\section{LITERATURE CITED}

Bertsekas DP, Tsitsiklis JN (2002) Introduction to probability. Athena Scientific, Nashua, NH

Carlotti F, Sciandra A (1989) Population dynamics model of Euterpina acutifrons (Copepoda: Harpacticoida) coupling individual growth and larval development. Mar Ecol Prog Ser 56:225-242

Editorial responsibility: Kenneth Sherman, Narragansett, Rhode Island, USA
Chen C, Beardsley RC, Cowles G (2006) An unstructured grid, finite-volume coastal ocean model (FVCOM) system. Oceanography 19(1):78-89

> Davis CS (1984a) Predatory control of copepod seasonal cycles on Georges Bank. Mar Biol 82:31-40

Davis CS (1984b) Interaction of copepod population with the mean circulation on Georges Bank. J Mar Res 42:573-590

Gaedke U (1990) Population dynamics of the calanoid copepods Eurytemora affinis and Acartia tonsa in the EmsDollart-Estuary: a numerical simulation. Arch Hydrobiol 118:185-226

GLOBEC (1992) Northwest Atlantic Implementation Plan. Report No. 6, US GLOBEC

> Gurney WSC, Speirs DC, Wood SN, Clarke ED, Heath MR (2001) Simulating spatially and physiologically structured populations. J Anim Ecol 70:881-894

Hu Q, Petrik CM, Davis CS (2007) Normal versus gamma: stochastic models of copepod molting rate. J Plankton Res 29(11):985-997

Longhurst AR, Reith AD, Bower RE, Seibert DLR (1966) A new system for the collection of multiple serial plankton samples. Deep-Sea Res 13:213-222

Miller CB, Tande KS (1993) Stage duration estimation for Calanus populations, a modelling study. Mar Ecol Prog Ser 102:15-34

Plagányi É, Hutchings L, Field JG, Verheye HM (1999) A model of copepod population dynamics in the Southern Benguela upwelling region. J Plankton Res 21:1691-1724

Runge JA, Franks PJS, Gentleman WC, Megrey BA, Rose KA, Werner FE, Zakardjian B (2004) Diagnosis and prediction of variability in secondary production and fish recruitment processes: developments in physical-biological modeling. In: Robinson AR, Brink K (eds) The sea, Vol 13. The global coastal ocean: multi-scale interdisciplinary processes. Harvard University Press, Cambridge, MA, p 413-474

Sciandra A (1986) Study and modelling of development of Euterpina acutifrons (Copepoda, Harpacticoida). J Plankton Res 8:1149-1162

> Souissi S, Ban S (2001) The consequences of individual variability in moulting probability and the aggregation of stages for modelling copepod population dynamics. J Plankton Res 23:1279-1296

Souissi S, Nival P (1997) Modeling of population dynamics of interacting species: effect of exploitation. Environ Model Assess 2:55-63

> Speirs DC, Gurney WSC, Holmes SJ, Heath MR and others (2004) Understanding demography in an advective environment: modelling Calanus finmarchicus in the Norwegian Sea. J Anim Ecol 73:897-910

Speirs DC, Gurney WSC, Heath MR, Wood SN (2005) Modelling the basin-scale demography of Calanus finmarchicus in the north-east Atlantic. Fish Oceanogr 14:333-358

Wroblewski JS (1980) A simulation of the distribution of Acartia clausi during Oregon Upwelling, August 1973. J Plankton Res 2:43-68

Submitted: March 15, 2007; Accepted: October 11, 2007

Proofs received from author(s): April 30, 2008 